\title{
Horizontal Transfer of Histone H3 by Mammalian Cells
}

Parker L. Sulkowski ${ }^{1,2}$, Hilary E. Nicholson ${ }^{1}$, Matthew Z. Li ${ }^{1}$, Norbert Perrimon ${ }^{2,3}$, Zhe Liu ${ }^{4}$, William G. Kaelin, Jr. 1,2,5*

${ }^{1}$ Department of Medical Oncology, Dana-Farber Cancer Institute, Harvard Medical School, Boston, MA, USA

${ }^{2}$ Howard Hughes Medical Institute, Chevy Chase, MD, USA.

${ }^{3}$ Department of Genetics, Blavatnik Institute, Harvard Medical School, Boston, MA, USA

${ }^{4}$ Janelia Research Campus, Howard Hughes Medical Institute, Ashburn, VA, USA

${ }^{5}$ Department of Medicine, Brigham and Women's Hospital, Harvard Medical School, Boston, MA, USA

*Correspondence should be addressed to William G. Kaelin, Jr., (William_Kaelin@dfci.harvard.edu) 


\begin{abstract}
Histones are small, highly basic, nuclear proteins that serve as structural elements to condense DNA into chromatin and regulate its accessibility ${ }^{1}$. Although histones released from dying cells can act as extracellular signaling molecules, all intracellular histone molecules are assumed to have originated from the cell in which they reside ${ }^{2-6}$. Here we show that histone $\mathrm{H3}$ is horizontally transferred between viable cells. Using an ER-targeted biotin ligase to detect secreted proteins $\mathrm{s}^{7,8}$, we serendipitously discovered that histone $\mathrm{H3}$ is selectively secreted by autophagic cells relative to histones $\mathrm{H} 2 \mathrm{~A}, \mathrm{H} 2 \mathrm{~B}$, and H4. Specific $\mathrm{H3}$ posttranslational modifications are enriched or depleted on secreted $\mathrm{H} 3$ relative to intracellular H3, suggesting that specific PTMs dictate H3's ability to be secreted or the information it conveys to neighboring cells. Remarkably, we found that secreted $\mathrm{H3}$ can enter the nuclei of recipient cells in an autophagy-independent and cell contactindependent manner ex vivo and in vivo. These findings have implications for cell-cell communication during nutrient deprivation, hypoxia, and perhaps other forms of cellular stress, and for the ability to deliver macromolecules across cell membranes.
\end{abstract}




\section{MAIN TEXT}

In hopes of identifying new secreted biomarkers controlled by the $V H L$ tumor suppressor gene, we introduced the BirA biotin ligase fused to an ER localization signal (ER-BirA ${ }^{7,8}$ ), or the corresponding empty expression vector (EV), into 786-O human $V H L$-/- clear cell renal carcinoma cells (ccRCCs) that reexpress the $V H L$ in the presence of Doxycycline (Dox) (Fig. 1a,b and Extended Data Fig. 1a,b). In pilot experiments we detected the secreted proteins IGFBP3, VEGF, and Clusterin in the conditioned media (CM) of the ER-BirA cells, but not the EV cells, after capture on streptavidin beads and immunoblot analysis (Fig. 1c). As expected from prior studies ${ }^{9}$, reexpression of VHL suppressed IGFBP3 and VEGF, and induced Clusterin. This assay was sensitive, based on the detection of VEGF (present at $<10 \mathrm{nM}$ in $\mathrm{CM}^{10,11}$ ) and specific, based on the failure to detect abundant intracellular proteins such as vinculin, $\beta$-Actin, $\beta$-Tubulin, COX IV, Golgin 97, Calreticulin and Lamin A/Cin the CM (Fig. 1c, d).

Surprisingly, we detected the apparent $K H L$-independent secretion of histone H3 (H3) with this assay (Fig. 1e). This was specific because histone H4 (H4), histone H2A (H2A), and histone H2B (H2B) were not detected in the CM. This was not a BirA artefact, because we likewise detected $\mathrm{H} 3$, but not $\mathrm{H} 4, \mathrm{H} 2 \mathrm{~A}$, or $\mathrm{H} 2 \mathrm{~B}$, in parental 786-O CM that was concentrated using a spin column or trichloroacetic acid (TCA) precipitation (Fig. 1f). No H3 was detected in unconditioned cell culture media (Extended Data Fig. 1c). In the experiments that follow, TCA precipitation was used to concentrate the CM unless otherwise specified. H3 secretion was confirmed using two independent $\mathrm{H} 3$ antibodies (Fig. 1g) and in multiple cell lines including RCC4, UMRC2, 769-P, and A498 ccRCC lines, MCF7 and MDA-MB-231 human breast carcinoma cells, U2OS human osteosarcoma cells, B16 mouse melanoma cells, and HOG human oligodendroglioma cells (Extended Data Fig. 1d-f). We also observed secretion of the oncohistone H3K27M $\mathrm{M}^{12,13}$ by SF8628 diffuse intrinsic pontine glioma cells (Extended Data Fig. 1f). The secreted $\mathrm{H} 3$ appeared to be monomeric, as determined by non-denaturing Native Gel Electrophoresis of spin column concentrated media (Extended Data Fig. 1g,h), and was sensitive to trypsin and proteinase $\mathrm{K}$ digestion, indicating that it was not encapsulated in an extracellular vesicle $^{14}$ (Extended Data Fig. 1i,j).

The specific detection of $\mathrm{H} 3$ without other histones (nor other abundant intracellular proteins) strongly argued against non-specific release of $\mathrm{H} 3$ by dying cells. Moreover, the posttranslational modifications of the secreted H3 were not representative of the total pool of H3, 
with a clear bias toward histone hypermethylation and against acetylation (Fig. 1h,i). Acetylation of H3 lysine 14 (H3K14) was an exception, as it was dramatically increased on secreted H3 relative to bulk H3 (Fig. 1h). This suggests that specific PTMs govern H3 secretion or its downstream consequences. In support of this idea, mutating H3 lysine 14 to arginine blocked secretion, whereas mutating it to glutamine to mimic lysine acetylation enhanced secretion (Fig. 1j), and mutating lysine 27 to methionine, corresponding to a canonical oncohistone mutation, increased H3 secretion (Extended Data Fig. 1k).

To ask how H3 is secreted, we treated RCC4 VHL-/- ccRCC cells with brefeldin A, which blocks canonical protein secretion ${ }^{15}$, or bafilomycin A1, which blocks autophagydependent secretion ${ }^{16,17}$. Brefeldin A blocked the secretion of IGFBP3, as expected, but did not block the secretion of $\mathrm{H} 3$ (Fig. 2a). In contrast, bafilomycin A1 blocked the secretion of H3, but not IGFB3(Fig. 2a), as did CRISPR/Cas9-based elimination of ATG7 or Beclin 1, which are required for autophagy ${ }^{17-19}$ (Fig. 2b-e).

Our CM experiments above were performed in synthetic, serum-free, media to reduce media complexity. Low serum promotes autophagy ${ }^{19,20}$. Notably, we did not detect apoptosis after 48 hours of serumestarvation (Extended Data Fig. 2a-f). Conversely, apoptosis-inducing agents did not promote the selective release of $\mathrm{H} 3$ (Extended Data Fig. 2g). We confirmed that readdition of serum to the culture medium suppressed $\mathrm{H} 3$ secretion (Fig. 2f), whereas induction of autophagy by treatment with rapamycin ${ }^{21}$ or exposure to hypoxia ${ }^{22}$ promoted $\mathrm{H} 3$ secretion (Fig. 2g-i and Extended Data Fig. 2h). Therefore, H3 undergoes autophagy-dependent secretion. Prompted by this finding, we confirmed that LC3B, which is well known to undergo autophagydependent secretion, was likewise captured in streptavidin pulldowns of CM from BirA-ER expressing cells (Extended data Fig. 2i) and that its secretion was likewise suppressed by bafilomycin A1, but not brefeldin A (Extended Data Fig. 2j).

To monitor the fate of secreted H3, we created 786-O cells that express $\mathrm{H} 3$ fused to mEmerald, a brightly fluorescent monomeric variant of green fluorescent protein, or mCherry, mixed them 1:1, grew them in serum-poor media, and performed live cell FACS. Remarkably, double-positive mEmerald/mCherry cells appeared within 48 hours (Fig. 3a, b and Extended Data Fig. 3a). This was specific, because double-positive cells were virtually absent when H2A, H2B, or H4 fusion cells, were tested in parallel (Fig. 3a, b and Extended Data Fig. 3a). Similar results were observed using a Doxycycline-inducible expression system in 786-O and $293 \mathrm{~T}$ cells 
(Extended Data Fig. 3 b-f) and when autophagy was stimulated by hypoxia rather than by growth in low serum (Extended Data Fig. 4a,b).

In a complementary set of experiments, we co-cultured $293 \mathrm{~T}$ human embryonic kidney cells expressing either H3-mCherry or H4-mCherry with RCC4 cells expressing a membranebound GFP (myr-GFP). Once again, we observed double-positive cells by FACS with the fluorescent $\mathrm{H} 3$ fusion protein cells, but not the H4 fusion protein cells (Fig. 3c,d). Using live cell fluorescence microscopy, we observed rare green cells with red nuclear inclusions that corresponded to mitotic chromosomes after Hoechst dye staining (Fig. 3c). Importantly, uptake of H3, in contrast to secretion of H3, was unaffected by loss of ATG7 or Beclin 1 (Extended Data Fig. 5a-d).

The apparent transfer of $\mathrm{H} 3$ did not require contact between donor and recipient cells based on transfer experiments in which filtered donor-conditioned media was added to separately plated recipient cells (Extended Data Fig. 5a-d) and based on transwell experiments in which recipient and donor cells were separated by a $3 \mu \mathrm{m}$ filter (Fig. 3e,f). To complement our fluorescence-based assays, we harvested the CM from 786-O cells expressing FLAG epitopetagged $\mathrm{H} 3$ or $\mathrm{H} 4$ andadded it to 786-O cells expressing BirA (Turbo-ID Variant ${ }^{7}$ ) fused to a nuclear localization signal (NLS) or the corresponding EV. 48 hours later we lysed the recipient cells, captured proteins on streptavidin beads, and did immunoblot assays. We detected Flag-H3, but not Flag-H4 in the streptavidin pulldowns from BirA-NLS extracts, but not EV extracts (Fig. $3 \mathrm{~g}, \mathrm{~h})$. As additional controls, we confirmed that abundant non-nuclear recipient cell proteins were not biotinylated in this assay (Fig. 3h).

In analogous experiments with SF8628 “donor” cells, which endogenously express H3K27M (see above), and 786-O cells “recipient" expressing BirA-NLS, we detected H3K27M in the streptavidin pulldowns from BirA-NLS extracts, but not EV extracts (Extended Data Fig. 5e,f), indicating endogenously produced oncohistones can be transferred to non-oncohistone producing cells.

Single-molecule, live-cell imaging of 293T cells expressing of $\mathrm{H} 3$ or $\mathrm{H} 4$ fused to HaloTag confirmed secretion of H3, but not H4, after serum-starvation (Extended Data Fig. 6a,b) The low basal H3-HaloTag and H4-HaloTag detected in the media without serum starvation by this highly sensitive technique may reflect the low basal levels of amphisomal secretion of nucleosomes reported in unstressed cells ${ }^{23}$. We co-cultured 293T cells expressing H3-HaloTag or 
H4-HaloTag with RCC4 cells expressing H2B-mEmerald in the absence of serum. 48 hours later, $15-20 \%$ of the H2B-mEmerald RCC4 cells contained H3-HaloTag, as determined by single molecule imaging, with $\sim 80 \%$ of the protein in the cytoplasm and $\sim 20 \%$ in the nucleus (Supplementary Movie 1, and Extended Data Fig. 6c-g). In contrast, transfer of H4 was virtually undetectable. It is possible that uptake into the nucleus would increase upon dissolution of the nuclear membrane during mitosis.

Encouraged by these results, we cocultured 786-O cells expressing $\mathrm{H} 3$ or $\mathrm{H} 4$ fused to Cre recombinase with mouse tex.loxP.EG cells ${ }^{24}$ that conditionally express GFP after Cre-mediated excision of a Lox-Stop-Lox cassette (Fig. 3i and Extended Data Fig. 7a). In pilot experiments we first confirmed that both the H3-Cre and H4-Cre fusions could recombine a GFP-based reporter in cotransfection experiments (Extended Data Fig. 7b-c). In contrast, only H3-Cre, but not H4$\mathrm{Cre}$, induced GFP expression in the recipient cell coculture experiments, arguing that $\mathrm{H} 3$, but not H4, can deliver a functional payload to the nucleus (Fig. 3j,k). Similar results, albeit with a lower recombination frequency, were obtained with a "color switch reporter ${ }^{25}$ " in which Crerecombination switches expression from a red fluorescent protein (RFP) to GFP (Fig. 31-n).

Consistent with the secretion results (Fig. 1j), mutating H3K14 to arginine blocked transfer, whereas mutating H3K14 to glutamine to mimic lysine acetylation enhanced transfer of both H3-Cre and fluorescently-tagged Histone 3 (Extended Data Fig. 7 and Extended Data Fig. $8)$.

To ask whether horizontal transfer of $\mathrm{H} 3$ can take place in vivo, we mixed 786-O cells expressing H3-Cre or H4-Cre 1:1 with 786-O cells with the color switch reporter and grew them subcutaneously in nude mice (Fig. 4a). Notably, the Cre expressing cells grew more slowly than the color switch cells, presumably because targeting Cre recombinase to chromatin impairs cellular fitness. Five weeks later, we removed palpable tumors, dissociated the tumor cells, and monitored recombination by FACS and immunoblot analysis for GFP-positive cells. The H3Cre, but not H4-Cre, cells promoted recombination of the color switch reporter (Fig. 4b-d, Extended Data Fig. 9a). Remarkably, H3-Cre-dependent recombination was also detectable when the Cre donor cells and color switch recipient cells were grown as separate tumors on opposite flanks (Fig. 4e-h, Extended Data Fig. 9b). Finally, we detected circulating H3, but not circulating H4, in non-tumor bearing mice that were fasted to promote autophagy ${ }^{26,27}$ (Fig. 4i,j). 
Histones released by dying cells can elicit danger signals by interacting with Toll-like receptors on cell surfaces and contribute to the pathogenesis of diseases such as septic shock ${ }^{2,5}$. Our findings indicate that autophagy causes the specific secretion of $\mathrm{H} 3$ in a form that can cross cell membranes and enter the nuclei of other cells. Our preliminary data indicate that certain PTMs are enriched or depleted on secreted H3 compared to the bulk H3 population, suggesting that specific PTMs influence H3 secretion, uptake, or the information conveyed to recipient cells. H3K14 acetylation, for example, appears to promote H3 secretion. Whether the transfer of H3 from donor to recipient cells influences cell behavior, perhaps through incorporation into chromatin or by a non-canonical function, remains to be determined. Transfer of histone H3 might reflect an ancient signaling mechanism that predated the emergence of cytokines, growth factors and hormones.

Solid tumors invariably contain hypoxic cells and are therefore likely to secrete H3. It will be important to ask if circulating $\mathrm{H} 3$ contributes to any of the systemic manifestations of cancer, such as cachexia and hypercoagulability, and whether they can serve as diagnostic or prognostic biomarkers. Our finding that an oncohistone can cross cell membranes also suggests that certain oncohistones play paracrine roles in transformation.

It has been known since the 1960s that exogenous histones can cross cell membranes under certain experimental conditions and can enhance the transfection efficiency of DNA, although the physiological relevance of these findings was previously unclear ${ }^{28-33}$. Delivering macromolecules across cell membranes has been a holy grail in drug discovery, although previous protein transduction methods based on positively charged peptides and proteins such as the Antennapedia protein homeodomain (penetratin), HIV TAT, and histones, have not proven robust $^{34}$. Our finding that the $\mathrm{H} 3$ secreted by autophagic cells can be used to deliver a protein cargo suggests that histone-based protein transduction can be optimized further by understanding the structure-function relationships underlying this phenomenon, including the importance of specific H3 PTMs, together perhaps with directed evolution. 


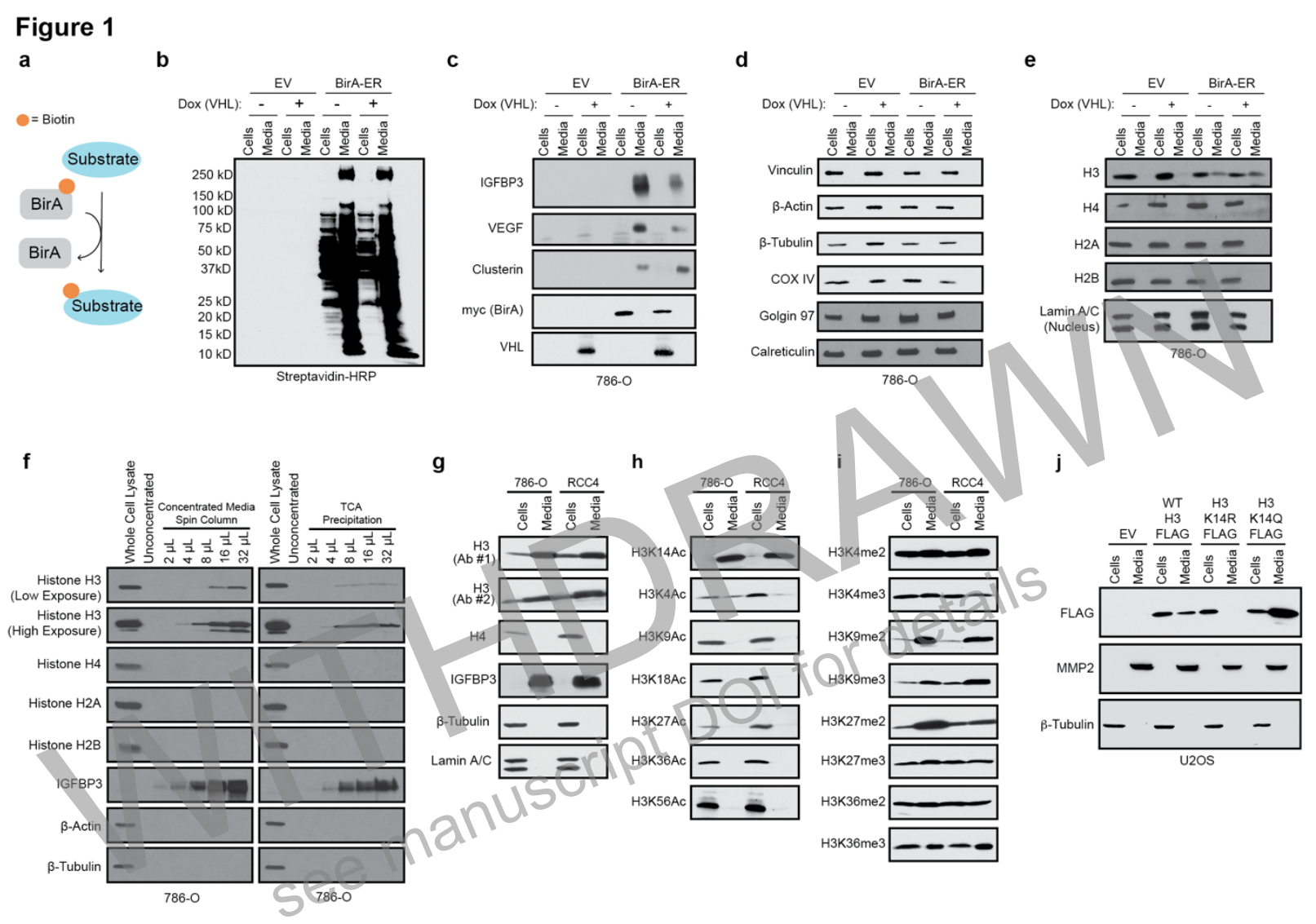

Fig. 1. Specific Secretion of Modified Histone H3

(a) Schema for protein biotinylation by BirA. (b-e). 786-O VHL-/- renal carcinoma cells expressing BirA-ER or the corresponding empty vector (EV) were grown, where indicated, in the presence of $2 \mu \mathrm{g} / \mathrm{mL}$ doxycycline (Dox) for 4 days to induce exogenous VHL expression prior to the addition of $50 \mu \mathrm{M}$ biotin. Cell lysates (Cells) and secreted proteins captured on streptavidin beads (Media) were resolved by SDS-PAGE, transferred to nitrocellulose, and probed with horseradish peroxidase-conjugated streptavidin (b) or with the indicated antibodies (c-e). (f) Immunoblot analysis of increasing amounts of 786-O cell conditioned media after concentration with a spin-column or by TCA precipitation compared to whole cell extract and unconcentrated media. (g-j) Immunoblot analysis of 786-O (g-i), RCC4 VHL-/- renal carcinoma (g-i), and U2OS $V H L+/+$ osteosarcoma cell (j) extracts and TCA-precipitated conditioned media. In (j) the cells expressed the indicated FLAG-tagged histone H3 point-mutants. 
Figure 2

a

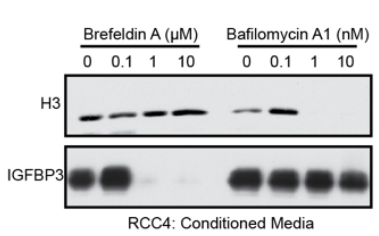

f

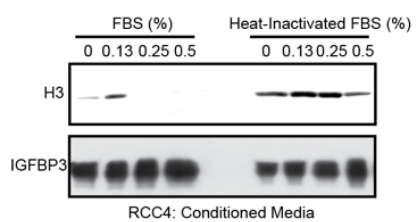

b

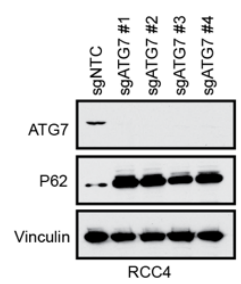

g c

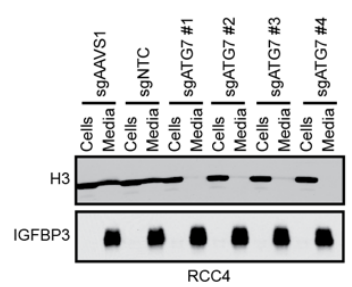

h

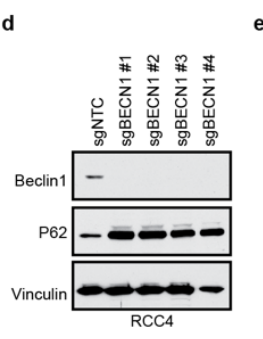

e

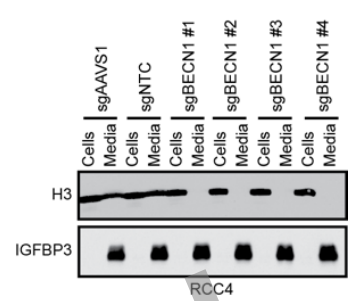

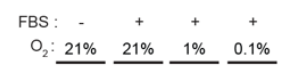

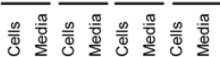

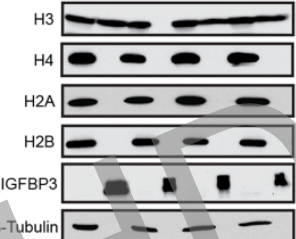

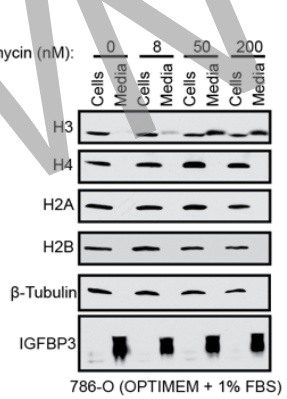

Fig. 2. Secretion of Histone $\mathrm{H} 3$ is Autophagy-Dependent

(a, f and $\mathbf{g}$ ). Immunoblot analysis of RCC4 cell extract and TCA precipitated conditioned media after the indicated treatments for $24 \mathrm{~h}$. (b-e) Immunoblot analysis of RCC4 cells after CRISPR/Cas9-based editing with the indicated sgRNAs (NTC is Nontargeting Control). Cells = whole cell extracts. Media = TCA precipitated conditioned media. (h and i) Immunoblot analysis of 786-O cells grown in Opti-MEM synthetic media supplemented with 1\% fetal bovine serum and the indicated amount of rapamycin for $24 \mathrm{~h}$, or grown under the indicated oxygen concentration. Cells $=$ whole cell extracts. Media $=$ TCA precipitated conditioned media . 
bioRxiv preprint doi: https://doi.org/10.1101/2021.06.30.450524; this version posted June 30, 2021. The copyright holder for this preprint (which was not certified by peer review) is the author/funder. All rights reserved. No reuse allowed without permission.

Figure 3

a

Co-Culture Experiments:

$\mathrm{mEmerald}$ Fusion Protein + mCherry Fusion Protein
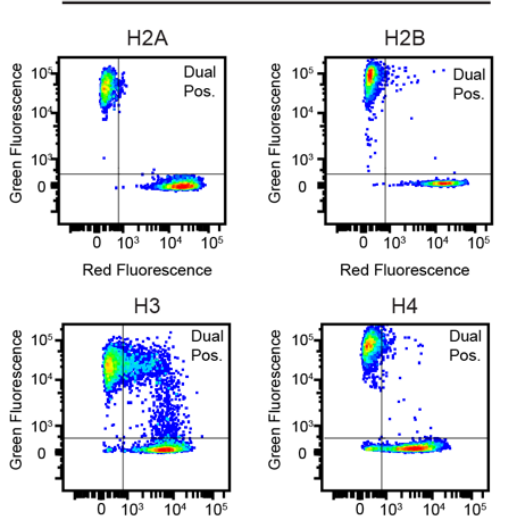

Red Fluorescence

Red Fluorescence

e

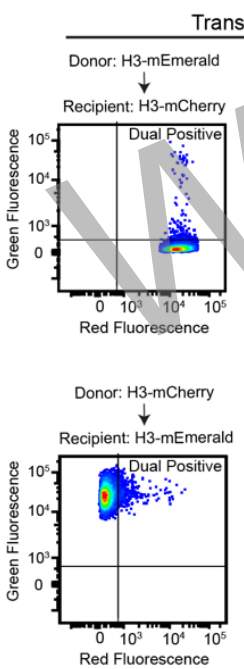

Transwell Assays

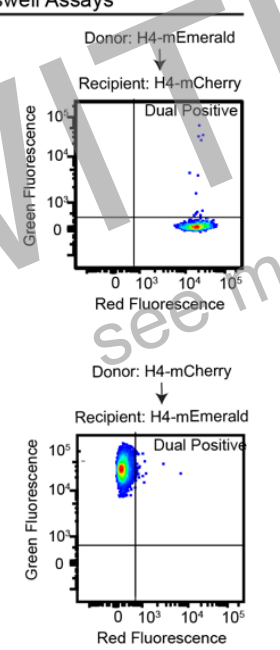

i

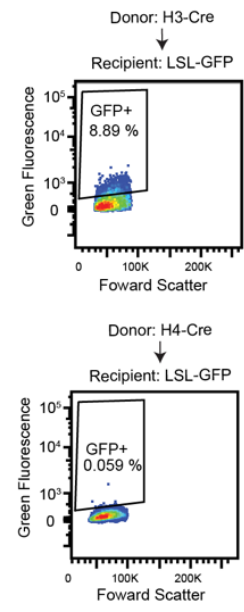

Lox-Stop-Lox GFP Cre Reporter

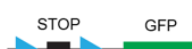

loxp loxP

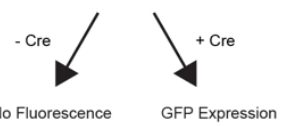

b

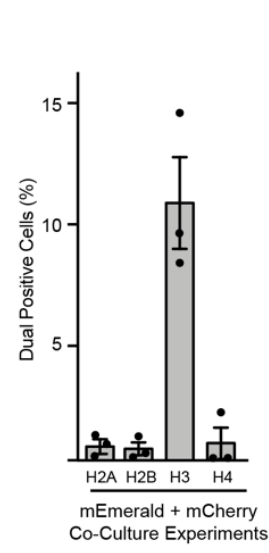

c

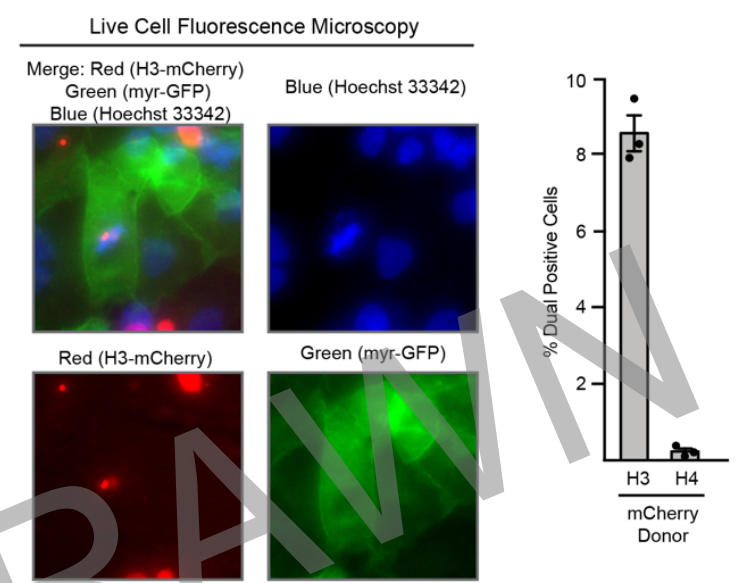

f

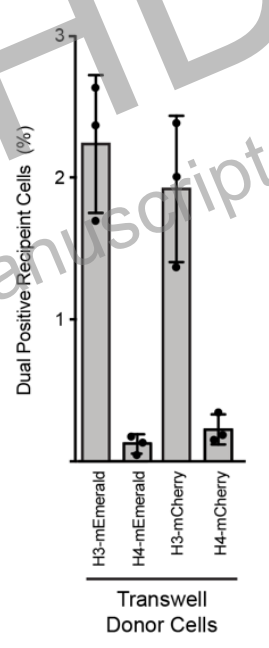

k

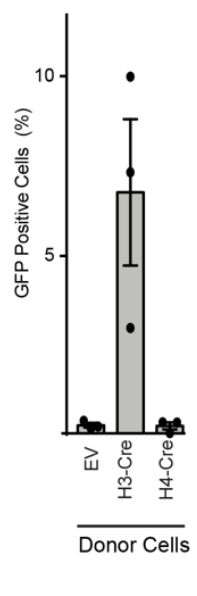

I

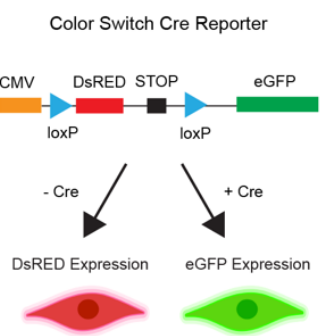

h
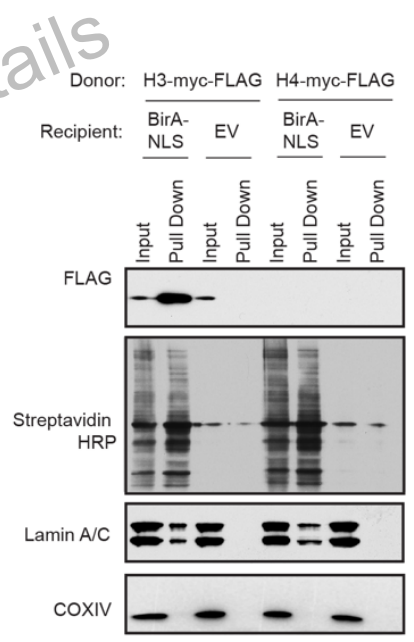

g

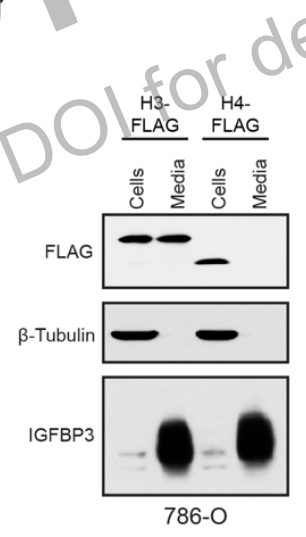




\section{Fig. 3. Horizontal Transfer of Histone H3 Ex Vivo}

(a and b). Raw data (a) and quantification (b) of 2 color FACS after co-culturing 786-O cells expressing the indicated histone fused to either mEmerald or mCherry (1:1 mix) in serum-free Opti-MEM for 2 days. (c and d). Representative live cell fluorescence micrographs (c) and quantification (d) after coculturing 293T embryonic kidney cells expressing either H3-mCherry or H4-mCherry with RCC4 cells expressing Myr-GFP in serum-free media for 2 days. (e and f). Raw data (e) and quantification (f) of 2 color FACS of recipient cells after growing the indicated donor and recipient 786-O cells in transwell plates in serum-free media for 2 days. (g) Immunoblot analysis of cell extract and TCA precipitated media from 786-O cells expressing either H3-FLAG or H4-FLAG. (h) 786-O cells expressing either BirA-NLS or EV were treated with conditioned media from 786-O cells expressing either H3-FLAG or H4-FLAG for 2 days. Cell lysates before (input) and after (pull down) capture on streptavidin beads were resolved by SDS-PAGE, transferred to nitrocellulose, and probed with horseradish peroxidase-conjugated streptavidin or with the indicated antibodies (i). Schematic for Cre recombinase reporter in LoxStop-Lox (L-S-L) GFP mouse Tcells. (j-k) Raw data (j) and quantification (k) after coculture of H3-Cre or H4-Cre 786-O cells with L-S-L-GFP cells (l). Schematic for Color Switch Cre recombinase reporter in 786-O cells. (m-n) Raw data (m) and quantification (n) after coculture of H3-Cre or H4-Cre 786-O cells with Color Switch reporter cells. For b,d,f,k, and n, data are mean \pm s.e.m. from $n=3$ biological replicates. 


\section{Figure 4}

a

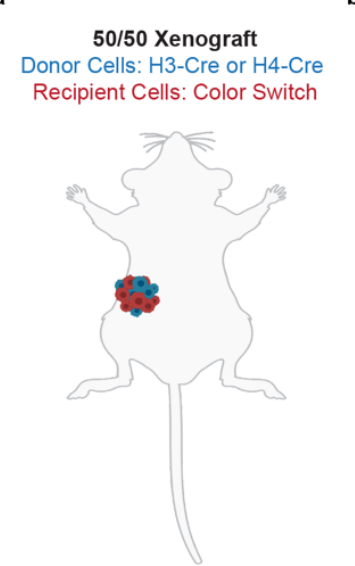

b
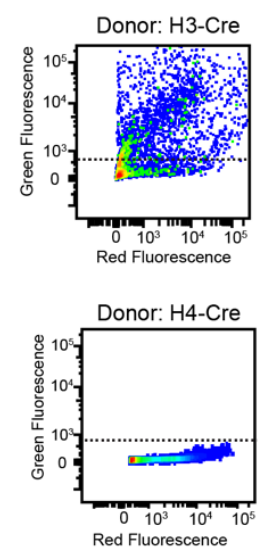

f

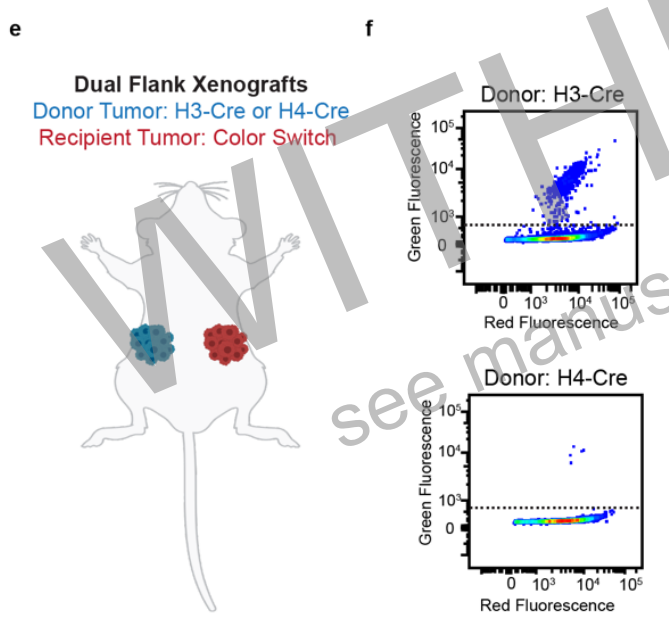

e

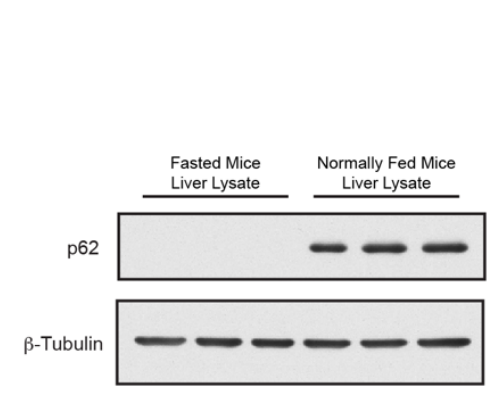

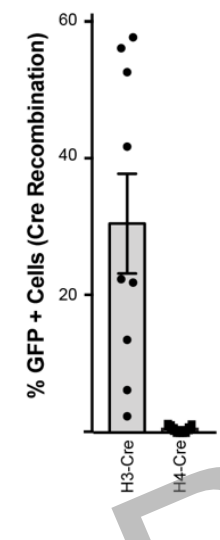
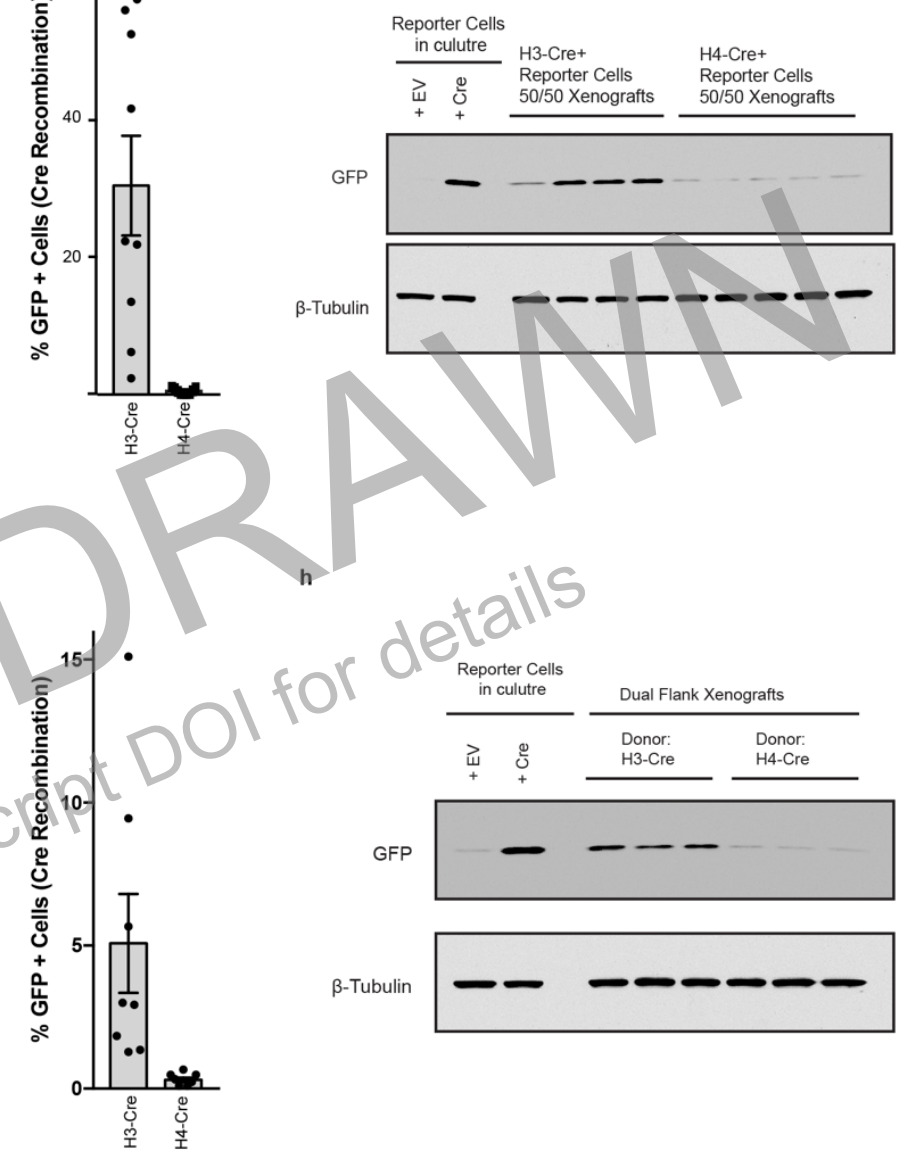

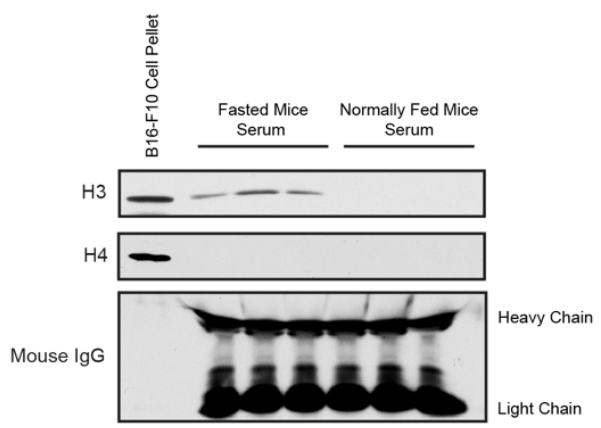

\section{Fig 4. Horizontal Transfer of Histone H3 In Vivo}

(a and e). Schemas for in vivo transfer experiments in tumor-bearing nude mice. Raw data (b) and quantification (c) of 2 color FACS of dissociated tumors formed 5 weeks after implanting 786-O cells expressing either H3-Cre or H4 Cre mixed 1:1 with RCC4 color switch reporter cells. (d) Immunoblot analysis of tumors from (a-c). 786-O color switch reporter cells infected 
ex vivo with a Cre expressing lentivirus (+Cre) or the empty vector $(\mathrm{EV})$ were included as controls. (f and $\mathbf{g}$ ) Raw data (f) and quantification (g) of 2 color FACS of tumors formed 786-O color switch reporter cells 5 weeks after implanting them on one flank and 786-O cells expressing either H3-Cre or H4 Cre on the opposite flank. (h) Immunoblot analysis of tumors from (e-g). 786-O color switch reporter cells infected ex vivo with a Cre expressing lentivirus $(+\mathrm{Cre})$ or the empty vector $(\mathrm{EV})$ were included as controls. (i-j) Immunoblot analysis of liver lysates (i) and serum (j) from c57/B16 mice that were or were not fasted for $24 \mathrm{~h}$. Each lane contains the same from a different mouse. For $\mathbf{c}$ and $\mathbf{g}$, each dot represents an independent tumor, bars are mean \pm s.e.m. 


\section{Acknowledgments}

We thank Steve Dowdy, Paul Mischel, Leon Murphy, and members of the Kaelin Laboratory for useful suggestions and Steve Dowdy, Cigal Kadoch, Peter Ratcliffe, and Peter Glazer for reagents. We thank Lixia He for her assistance. P. L.S is a Howard Hughes Medical Institute Fellow of the Damon Runyon Cancer Research Foundation, DRG- 2430-21. W.G.K. is supported by an NIH R35CA210068 and NIH P50CA101942 and is an HHMI Investigator.

\section{Author contributions}

P.L.S. designed and performed experiments and contributed to all aspects of the study. H.E.N. performed experiments cloning and developing BirA*_G3-ER system in 786-O cells. M.Z.L. performed experiments and contributed to data analysis and compiling of the manuscript. N.P. provided reagents and introduced the BirA system to the Kaelin Laboratory. Z.L. designed, performed, and analyzed the Single-molecule imaging studies, and contributed to compiling of the manuscript. W.G.K. conceptualized and supervised the study and interpreted the data. W.G.K., and P.L.S. wrote the manuscript.

\section{Competing interests}

W.G.K., and P.L.S. are inventors on the U.S. patent application submitted by and assigned to Dana-Farber Cancer Institute, which covers Recombinant Histone Polypeptides and Uses Thereof. W.G.K. has financial interests in Circle Pharma, LifeMine Therapeutics, Lilly Pharmaceuticals, Fibrogen, Cedilla Therapeutics, Nextech Invest, Tango Therapeutics.

\section{Data Availability}

All data generated or analysed during this study are included in this published article (and its supplementary information files) There are no restrictions on data availability. All unique biological materials will be available. Please contact W.G.K., the corresponding author.

\section{Code Availability Statement}

There was no custom code used in this study. 


\section{References}

1 Jenuwein, T. \& Allis, C. D. Translating the histone code. Science 293, 1074-1080 (2001).

$2 \mathrm{Xu}$, J. et al. Extracellular histones are major mediators of death in sepsis. Nature medicine 15, 1318-1321 (2009).

3 Remijsen, Q. et al. Dying for a cause: NETosis, mechanisms behind an antimicrobial cell death modality. Cell Death \& Differentiation 18, 581-588 (2011).

4 Yipp, B. G. \& Kubes, P. NETosis: how vital is it? Blood 122, 2784-2794 (2013).

5 Silk, E., Zhao, H., Weng, H. \& Ma, D. The role of extracellular histone in organ injury. Cell death \& disease 8, e2812-e2812 (2017).

6 Bernardes, N. E. \& Chook, Y. M. Nuclear import of histones. Biochemical Society Transactions 48, 2753-2767 (2020).

7 Branon, T. C. et al. Efficient proximity labeling in living cells and organisms with TurboID. Nature biotechnology 36, 880-887 (2018).

8 Droujinine, I. A. et al. Proteomics of protein trafficking by in vivo tissue-specific labeling. Nature Communications 12, 2382, doi.10.1038/s41467-021-22599-x (2021).

9 Nakamura, E. et al. Clusterin is a secreted marker for a hypoxia-inducible factorindependent function of the von Hippel-Lindau tumor suppressor protein. The American journal of pathology 168, 574-584 (2006).

10 Iliopoulos, O., Levy, A.P., Jiang, C., Kaelin, W. G. \& Goldberg, M. A. Negative regulation of hypoxia-inducible genes by the von Hippel-Lindau protein. Proceedings of the National Academy of Sciences 93, 10595-10599 (1996).

11 Siemeister, G. et al. Reversion of deregulated expression of vascular endothelial growth factor in human renal carcinoma cells by von Hippel-Lindau tumor suppressor protein. Cancer Research 56, 2299-2301 (1996).

12 Nacev, B. A. et al. The expanding landscape of 'oncohistone'mutations in human cancers. Nature 567, 473-478 (2019).

13 Koch, L. Cancer genetics: Oncohistone pathology explained. Nat Rev Genet 17, 375, doi:10.1038/nrg.2016.71 (2016).

14 Leidal, A. M. et al. The LC3-conjugation machinery specifies the loading of RNAbinding proteins into extracellular vesicles. Nature cell biology 22, 187-199 (2020).

15 Donaldson, J. G., Finazzi, D. \& Klausner, R. D. Brefeldin A inhibits Golgi membranecatalysed exchange of guanine nucleotide onto ARF protein. Nature 360, 350-352 (1992).

16 Yoshimori, T., Yamamoto, A., Moriyama, Y., Futai, M. \& Tashiro, Y. Bafilomycin A1, a specific inhibitor of vacuolar-type $\mathrm{H}(+)$-ATPase, inhibits acidification and protein degradation in lysosomes of cultured cells. Journal of Biological Chemistry 266, 1770717712 (1991).

17 Cavalli, G. \& Cenci, S. Autophagy and protein secretion. Journal of Molecular Biology 432, 2525-2545 (2020).

18 Yu, L. et al. Regulation of an ATG7-beclin 1 program of autophagic cell death by caspase-8. Science 304, 1500-1502 (2004).

19 Mizushima, N. Autophagy: process and function. Genes \& development 21, 2861-2873 (2007). 
20 Kaizuka, T. et al. An autophagic flux probe that releases an internal control. Molecular cell 64, 835-849 (2016).

21 Jung, C. H., Ro, S.-H., Cao, J., Otto, N. M. \& Kim, D.-H. mTOR regulation of autophagy. FEBS letters 584, 1287-1295 (2010).

22 Mazure, N. M. \& Pouysségur, J. Hypoxia-induced autophagy: cell death or cell survival? Current opinion in cell biology 22, 177-180 (2010).

23 Jeppesen, D. K. et al. Reassessment of exosome composition. Cell 177, 428-445. e418 (2019).

24 Wadia, J. S., Stan, R. V. \& Dowdy, S. F. Transducible TAT-HA fusogenic peptide enhances escape of TAT-fusion proteins after lipid raft macropinocytosis. Nature medicine 10, 310-315 (2004).

25 Zomer, A. et al. In vivo imaging reveals extracellular vesicle-mediated phenocopying of metastatic behavior. Cell 161, 1046-1057 (2015).

26 Komatsu, M. et al. Impairment of starvation-induced and constitutive autophagy in Atg7deficient mice. Journal of Cell Biology 169, 425-434 (2005).

27 Mizushima, N., Yamamoto, A., Matsui, M., Yoshimori, T. \& Ohsumi, Y. In vivo analysis of autophagy in response to nutrient starvation using transgenic mice expressing a fluorescent autophagosome marker. Molecular biology of the cell 15, 1101-1111 (2004).

28 Hariton-Gazal, E., Rosenbluh, J., Graessmann, A., Gilon, C. \& Loyter, A. Direct translocation of histone molecules across cell membranes. Journal of Cell Science 116, 4577-4586 (2003).

29 Fritz, J.D., Herweijer, H,Zhang, G. \& Wolff, J. A. Gene transfer into mammalian cells using histone-condensed plasmid DNA. Human gene therapy 7, 1395-1404 (1996).

30 Ryser, H. J.-P \& Hancock, R. Histones and basic polyamino acids stimulate the uptake of albumin by tumor cells in culture. Science 150, 501-503 (1965).

31 Schwartz, A. The Effects of Histones and Other Polycations on Cellular Energetics: I. MITOCHONDRIAL OXIDATIVE PHOSPHORYLATION. Journal of Biological Chemistry 240, 939-943 (1965).

32 Han, H. et al. A comprehensive review on histone-mediated transfection for gene therapy. Biotechnology advances 37, 132-144 (2019).

33 Wagstaff, K. M., Glover, D. J., Tremethick, D. J. \& Jans, D. A. Histone-mediated transduction as an efficient means for gene delivery. Molecular Therapy 15, 721-731 (2007).

34 Lönn, P. \& Dowdy, S. F. Cationic PTD/CPP-mediated macromolecular delivery: charging into the cell. Expert opinion on drug delivery 12, 1627-1636 (2015). 